\title{
Pattern dynamics of vortex ripples in sand: Nonlinear modeling and experimental validation
}

Andersen, Ken Haste; Abel, M.; Krug, J.; Ellegaard, C.; Søndergaard, L. R.; Udesen, J.

Published in:

Physical Review Letters

Link to article, DOI:

10.1103/PhysRevLett.88.234302

Publication date:

2002

Document Version

Publisher's PDF, also known as Version of record

Link back to DTU Orbit

Citation (APA):

Andersen, K. H., Abel, M., Krug, J., Ellegaard, C., Søndergaard, L. R., \& Udesen, J. (2002). Pattern dynamics of vortex ripples in sand: Nonlinear modeling and experimental validation. Physical Review Letters, 88(23), 234302. https://doi.org/10.1103/PhysRevLett.88.234302

\section{General rights}

Copyright and moral rights for the publications made accessible in the public portal are retained by the authors and/or other copyright owners and it is a condition of accessing publications that users recognise and abide by the legal requirements associated with these rights.

- Users may download and print one copy of any publication from the public portal for the purpose of private study or research.

- You may not further distribute the material or use it for any profit-making activity or commercial gain

- You may freely distribute the URL identifying the publication in the public portal 


\title{
Pattern Dynamics of Vortex Ripples in Sand: Nonlinear Modeling and Experimental Validation
}

\author{
K. H. Andersen, ${ }^{1}$ M. Abel, ${ }^{2}$ J. Krug, ${ }^{3}$ C. Ellegaard, ${ }^{4}$ L. R. Søndergaard,,${ }^{4,5}$ and J. Udesen ${ }^{4,5}$ \\ ${ }^{1}$ Department of Mechanical Engineering, Technical University of Denmark, DK-2800 Kgs. Lyngby, Denmark \\ ${ }^{2}$ Institut für Physik, Universität Potsdam, D-14415 Potsdam, Germany \\ ${ }^{3}$ Fachbereich Physik, Universität Essen, D-45117 Essen, Germany \\ ${ }^{4}$ Niels Bohr Institute, University of Copenhagen, DK-2100 Copenhagen Ø, Denmark \\ ${ }^{5}$ Department of Mathematics and Physics, University of Roskilde, Box 260, DK-4000 Roskilde, Denmark
}

(Received 30 January 2002; published 22 May 2002)

\begin{abstract}
Vortex ripples in sand are studied experimentally in a one-dimensional setup with periodic boundary conditions. The nonlinear evolution, far from the onset of instability, is analyzed in the framework of a simple model developed for homogeneous patterns. The interaction function describing the mass transport between neighboring ripples is extracted from experimental runs using a recently proposed method for data analysis, and the predictions of the model are compared to the experiment. An analytic explanation of the wavelength selection mechanism in the model is provided, and the width of the stable band of ripples is measured.
\end{abstract}

DOI: 10.1103/PhysRevLett.88.234302

PACS numbers: 45.70.Qj, 47.20.Lz, 47.54.+r

Ever since the establishment of a conceptual framework for pattern formation [1], the description of patterns formed in sand by the flow of wind or water has posed a challenge to the community [2-7]. Despite diverse efforts including coupled map models for ripples and dunes in air [2], stochastic models for ripples in air [3], or continuum equations based on the symmetries of the problem [4], theoretical understanding has remained sparse. For example, all models display a coarsening of the ripple/dune pattern, but the coarsening does not terminate at a final selected wavelength, as is frequently observed in nature. Furthermore, the models are heuristic, and it is not possible to make a quantitative comparison with experiments.

Here we study vortex ripples [8], which are created by an oscillatory water flow, such as that generated near the sand bed by a surface wave. Vortex ripples have attracted attention as an example of a nonlinear pattern forming system with a strongly subcritical first bifurcation $[6,7,9]$, which cannot be described by conventional methods like amplitude equations [9]. As most other sand patterns, they display coarsening and saturation at a finite wavelength. The approach pursued in this Letter combines a simple model for the fully developed pattern with a sophisticated data analysis which allows one to extract the key model ingredient - the interaction function $f(\lambda)$ - directly from the experimental runs. In this way the validity of the model can be tested, and additional features required for the description can be identified. As far as we know, this is the first quantitative comparison between theory and experiment for a sand pattern. The basic ideas are general, and we expect that the theoretical formalism combined with the experimental analysis can be used for related sand patterns (e.g., the one studied in [5]), or other strongly nonlinear systems.

One-dimensional vortex ripples can be created in an annular channel $[6,10]$, ensuring the pattern to be subject to well-defined periodic boundary conditions. Freely grown ripples are created from a flat bed by a coarsening process, which eventually saturates at an equilibrium state, where the ripple length $\tilde{\lambda}$ is almost independent of the frequency $\nu$ of the driving and proportional to the amplitude of the oscillation of the plate $a[8,11]$. Here we are mostly concerned with the stability and evolution of the ripple patterns themselves, and not with the instability of the flat bed, which has been discussed elsewhere $[9,12]$. By creating a homogeneous ripple pattern, where all ripples have the same length, and changing amplitude and frequency, the stability of the pattern is probed. In this way it was found that there is a stable band of ripples, $\lambda_{\min }<\tilde{\lambda}<\lambda_{\max }$, for a given set of driving conditions [7].

Recently, a simple model was proposed, which describes both the coarsening and saturation of ripples, and reproduces the existence of a stable band [9]. In its simplest form, the change of the length $\lambda_{j}$ of the ripple $j$ is a function of $\lambda_{j}$ itself and the lengths of the neighboring ripples:

$$
\dot{\lambda}_{j}=-f\left(\lambda_{j-1}\right)+2 f\left(\lambda_{j}\right)-f\left(\lambda_{j+1}\right) \text {. }
$$

The interaction function $f(\lambda)$ describes the transfer of mass, and consequently length, between neighboring ripples. Behind each ripple, due to the water oscillation, a separation vortex forms, which causes an exchange of mass between the ripples. $f\left(\lambda_{j}\right)$ is the amount of sand which is taken by a ripple of length $\lambda_{j}$ from its downstream neighbor during one-half of the oscillation (Fig. 1). At the same time ripple $j$ loses an amount of mass $f\left(\lambda_{j-1}\right)$ due to the action of the upstream ripple. Adding the reverse process for the second half of the oscillation results in the model (1) [9]. The purpose of this Letter is a test of this model through comparison with experimental data.

Experimental setup.-As shown in Fig. 2, an $11 \mathrm{~mm}$ wide, $15 \mathrm{~cm}$ high annular Plexiglas channel of diameter $48.6 \mathrm{~cm}$ is filled with water, $A$, and glass beads, $B$, with a diameter of $250 \pm 50 \mu \mathrm{m}$. In the middle is a conical 


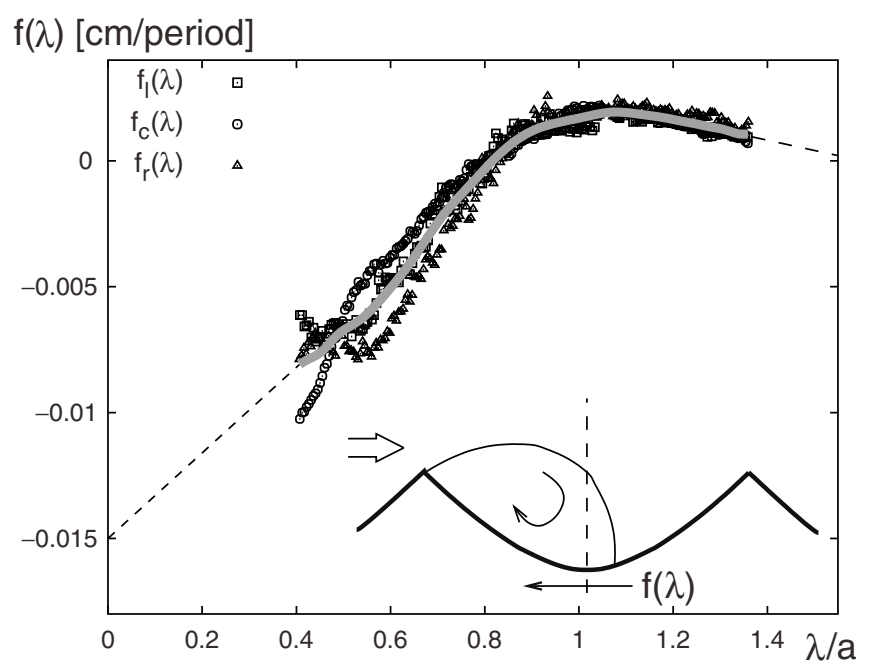

FIG. 1. The interaction function for $a=6 \mathrm{~cm}$ and $\nu=$ $0.6 \mathrm{~Hz}$. The thick gray line is the smooth average of the three different functions (mean values subtracted). The right limit of the plot at $\lambda / a=1.45$ is the limit of stability, where new ripples are created $\left(\lambda_{\max }\right)$. The inset shows a sketch of the ripples in the part of the oscillation when the flow is from the left to the right. The interaction function can be interpreted as the transport of sand in the trough, across the vertical dashed line.

mirror, $C$, which is filmed from above by a CCD camera with a resolution of $640 \times 480$ pixels. The channel is attached to a motor, $D$, by a $1.5 \mathrm{~m}$ long arm $E$, which oscillates the channel in an almost sinusoidal fashion.

We create initial conditions with small ripples by oscillating at a small amplitude. The experiment starts when amplitude and frequency are changed to their desired values. Throughout this paper, we use only the condition $a=6 \mathrm{~cm}, \nu=0.6 \mathrm{~Hz}$. We want to emphasize that there is nothing special about this condition, the same qualitative results are obtained for other parameters. Growing the ripples from initial conditions with homogeneous ripples smaller than $\lambda_{\min }$ results in a final number of 19-21 ripples with a length (averaged over 16 realizations) of $\tilde{\lambda} / a=1.25$. The uncertainty in the length due to

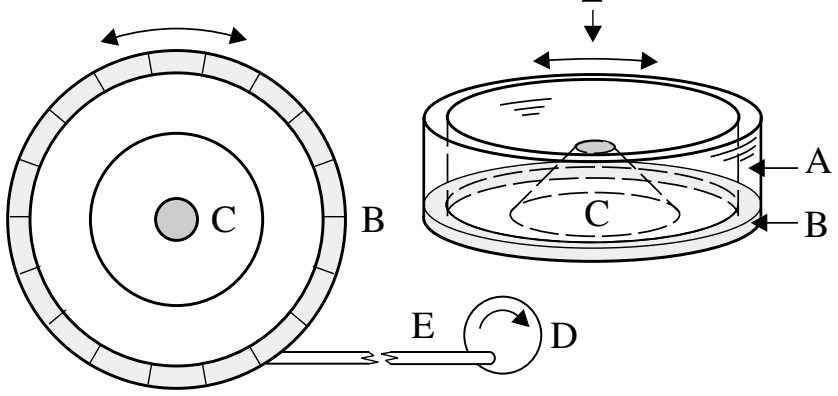

FIG. 2. A sketch of the experimental setup seen from above (left) and from the side (right). The length of the arm $E$ and the width of the channel are not to scale. See text. periodicity is around 5\%. The images are transformed back to Cartesian coordinates, and the lengths $\lambda_{j}$ are found by fitting them to triangles with fixed slope (Fig. 3a). From series of consecutive $\lambda_{j}$, the temporal change, $\dot{\lambda}_{j}$, is established, and a space-time plot of the ripple evolution is constructed. Figure $3 \mathrm{~b}$ shows the evolution starting from a small initial wavelength, which leads to the annihilation of ripples. Around each annihilation the ripples have not been analyzed, as it becomes inaccurate to fit the triangles during these events.

Measuring the stable band.-By running the experiment with different values of $a$ until a homogeneous pattern occurs, we create initial conditions with a different number $N$ of ripples. From this initial pattern, the experiment is started with the above parameters, and run for 10000 periods. We have created initial conditions with $N \in[17 ; 24]$ and observed that for $N>22$ ripples are annihilated, while for $N<18$ one or more new ripples are created. We therefore conclude that there exists a stable band $N \in[17.5 ; 22.5]$ (for $a=6 \mathrm{~cm}, \nu=0.6 \mathrm{~Hz}$ ), which corresponds to $\lambda_{\min } / a=1.13 \pm 0.03$ and $\lambda_{\max } / a=1.45 \pm 0.05$. Note that this is a more accurate measurement of the stable band than that conducted in

(a)

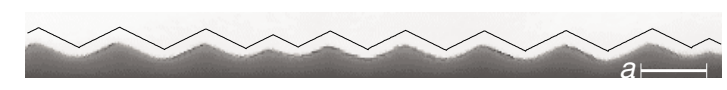

(b)
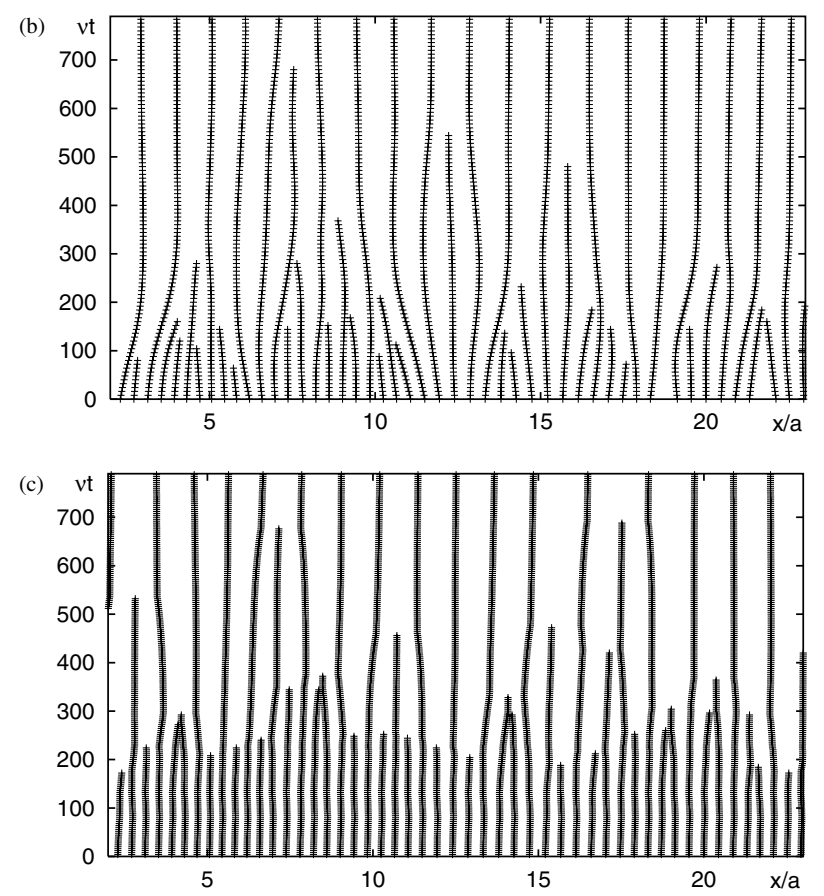

FIG. 3. (a) An example of an extracted profile (opaque region) and the fitted triangles with constant slope. The line is shown above the profile for clarity. (b) Space-time plot of the experimental evolution of the position of the ripple crests starting from ripples with lengths $2.5 \mathrm{~cm}$ and evolving with $a=6 \mathrm{~cm}$ and $\nu=0.6 \mathrm{~Hz}$. (c) A simulation of the model (1) using the extracted interaction function and the same initial conditions as above. 
$[6,7]$, as we are always forcing with the same values of $a$ and $\nu$.

Mass transfer model.-The model (1) was originally presented in Ref. [9], together with a more refined version. A stability analysis of the homogeneous state $\lambda_{j} \equiv \bar{\lambda}$ shows that ripples are stable (unstable) if the derivative $f^{\prime}(\bar{\lambda})<0(>0)$. For a convex interaction function, the lower stability boundary $\lambda_{\min }$ therefore lies at $\lambda_{\text {marg }}$, where $f^{\prime}\left(\lambda_{\text {marg }}\right)=0$. To investigate nonlinear pattern evolution within this model, Eq. (1) is supplemented by the rule that ripples which reach zero length are annihilated and removed from the system of equations, while the remaining ones are relabeled.

Simulations of the model starting from typical initial conditions [13] in the unstable band $\lambda<\lambda_{\text {marg }}$ show that an equilibrium wavelength is reached which is essentially independent of the initial wavelength, and depends only on the interaction function $f(\lambda)$. To gain some insight into the selection mechanism, it is useful to recast the model into potential form by writing it in terms of the position $x_{j}$ of the troughs between the ripples defined by $\lambda_{j}=x_{j+1}-x_{j}$. Then $\dot{x}_{j}=-\partial V / \partial x_{j}$, with the potential $V$ given by

$$
V=-\sum_{j=1}^{N} \int_{0}^{x_{j+1}-x_{j}} d \lambda f(\lambda),
$$

where $N$ is the number of ripples. It is then plausible to conjecture that the equilibrium length $\tilde{\lambda}$ can be found by minimizing $V$ for homogeneous ripples, under the constraint that the total length $L=N \tilde{\lambda}$ is conserved. This implies that $\tilde{\lambda}$ is determined through the Maxwell construction applied to $f$ :

$$
\int_{0}^{\tilde{\lambda}} d \lambda f(\lambda)=\tilde{\lambda} f(\tilde{\lambda}) .
$$

Comparison with numerical simulations shows that (3) systematically overestimates $\tilde{\lambda}$, with a better performance the steeper the stable branch of $f$. Our interpretation is that the deterministic dynamics gets stuck in the multitude of metastable states of (1); recall that any homogeneous state with $\bar{\lambda}>\lambda_{\text {marg }}$ is a stable, stationary solution. Since the mean ripple length can increase only by annihilations, its evolution freezes once all ripples are in the stable band. The wavelength predicted by (3) should therefore be an upper bound on the actual equilibrium wavelength, which is true in all cases we have considered.

Data analysis. - Given the time series $\lambda_{j}(t)$ we want to (i) evaluate how well the model (1) describes the evolution of the ripples and (ii) extract the interaction function $f(\lambda)$. For the analysis, we write (1) in the more general form

$$
\dot{\lambda}_{j}=-f_{l}\left(\lambda_{j-1}\right)+2 f_{c}\left(\lambda_{j}\right)-f_{r}\left(\lambda_{j+1}\right),
$$

where $f_{l}, f_{c}$, and $f_{r}$ denote the left neighbor, center, and right neighbor interaction function, respectively. This yields an additional degree of freedom as the functions are not required to be equal.
We want to determine the optimal transformations $f_{l}\left(\lambda_{j-1}\right), f_{c}\left(\lambda_{j}\right), f_{r}\left(\lambda_{j+1}\right)$ in the sense that they minimize the error $\chi^{2}=\sum_{t}\left[\dot{\lambda}_{j}(t)+f_{l}(t)-2 f_{c}(t)+f_{r}(t)\right]^{2}$. The problem is solved numerically by the alternating conditional expectation value algorithm [14,15]. The algorithm works by varying $f_{l, c, r}$ in the space of all measurable functions until convergence to the absolute minimum of $\chi^{2}$ [14]. The results are nonparametric functions which are given in numerical form by points, e.g., $\left[\lambda_{j}, f_{c}\left(\lambda_{j}\right)\right]$. An upper bound on the error, e.g., on $f_{c}$, is given by $\sigma_{f_{c}}=\max _{\lambda}\left(d f_{c} / d \lambda\right) \cdot \sigma_{\lambda}$, with $\sigma_{\lambda}$ being the measurement error at the point $\lambda$; analogous estimates apply to $f_{l}, f_{r}$. The error in the points on the $\lambda$ axis is estimated using the above result and equals roughly the errors in the given values of $\lambda$.

The quality of each of the resulting functions is given by the maximum correlation $\psi_{l, c, r}$ of one of the terms with the sum of all the others [16]. A value of $\psi=1$ implies a perfect result, lower values indicate either imperfect modeling or (measurement) noise or both.

Results. - We have performed the analysis on data from 18 realizations of a coarsening process initiated with approximately 60 small ripples and run with $a=6 \mathrm{~cm}$ and $\nu=0.6 \mathrm{~Hz}$. Figure 1 shows the three functions $f_{l}, f_{c}$, and $f_{r}$ averaged over the 18 runs. Clearly there is some noise, but the functions are very similar as expected from (1). The maximum correlations are found to be $\Psi_{l}=0.66$, $\Psi_{c}=0.88, \Psi_{r}=0.72$. The uncertainty in estimating $\lambda$ amounts to about $2 \mathrm{~mm}$, with a maximum slope of $f$ of around $3 \times 10^{-3}$ (period) $)^{-1}$, the absolute error of $f$ is $6 \times 10^{-4} \mathrm{~cm} /$ period. This means that the result does not fluctuate due to lack of data, rather the model (4) does not account for all the variation in the ripple evolution. We will return to this point later.

The maximum of the function lies around $\lambda_{\text {marg }}=$ $1.08 a$, which is a little larger than what was found by the numerical simulations in [9]. Concerning the shape of the function, it is interesting that the slope in the unstable band $\lambda<\lambda_{\text {marg }}$ is much larger than the slope in the stable band $\lambda>\lambda_{\text {marg. }}$. As the slope is a measure of the time scale of the dynamics, this implies that the initial coarsening stage is much faster than the equilibrating stage.

To use the resulting function to integrate (1) numerically for evaluating (3), information about the smallest ripples is needed. With a lack of such information, we have extrapolated linearly to $\lambda=0$, Fig. 1, dashed line. Varying the slope of the extrapolated part by a factor of 2 in either direction does not change the final number of ripples. Figure $3 \mathrm{c}$ shows a space-time plot of the numerical integration. The model predicts a qualitatively similar behavior as the experiment; however, the instability of the small ripples develops slower in the model than in the experiment. If the extrapolated slope for the interaction function is made steeper, this instability will evolve faster in the model. 
The model also overestimates the final ripple length. For similar initial conditions and system size, the final number of ripples is typically 18 , whereas the experiment yields 20.

Evaluating the Maxwell construction (3) using the measured function and extrapolating to account for the larger ripples, gives $\tilde{\lambda}=2.2 a$, which lies far outside of the range of definition of $f(\lambda)$. Evidently, the experimentally determined $f(\lambda)$ belongs to the class of interaction functions for which the upper bound given by Eq. (3) is not useful.

The upper bound of the stable band, where new ripples are created, was found to be $\lambda_{\max }=1.45 \mathrm{a}$. At this point, an infinitesimally small ripple, inserted in the trough between two larger ripples, is able to gain mass from the neighbors, and thus grows. If the model shall capture this behavior, Eq. (1) yields that $f\left(\lambda_{\max }\right)$ should be smaller than $f(0)$ [9]; otherwise the right-hand side is negative and a small ripple disappears. The measured interaction function (cf. Fig. 1), however, suggests the opposite. Even with the large uncertainty inherent in the extrapolation, a bend which makes $f(1.45 a)<f(0)$ is hard to imagine. We therefore conclude that the model in the form (1) is not able to quantitatively predict the creation of new ripples. The reason for this apparent failure relates to the model being developed essentially as an expansion around a homogeneous state [9]. But in the case of a creation, the state is as inhomogeneous as it can be: a very tiny ripple flanked by large ripples. To account for this, the interaction function should be described as a function of the length of the ripple creating the separation bubble, $\lambda_{i}$, but also of the length of its neighbors. Ongoing numerical work indicates that it is most relevant to write $f \equiv f\left(\lambda_{i}, \lambda_{i+1}\right)$ where $\lambda_{i}$ is the length of the ripples creating the separation bubble, and $\lambda_{i+1}$ is the one "touched" by the separation bubble (see inset of Fig. 1). In fact, the whole coarsening process is dominated by highly inhomogeneous configurations, and this might also be why simulating (1), using the measured interaction function, does not produce exactly the correct final ripple length.

Conclusions. - We have demonstrated that we can extract the interaction function $f(\lambda)$ from spatiotemporal data of the evolution of the profile of the sand surface. The nonlinear data analysis shows its full strength, producing nonparametric function estimates, where any parametric approach would have failed. With the interaction function it is possible to model the evolution of the ripples, in particular, their coarsening and equilibration.

The prediction from the model of the existence of a stable band is indeed observed in the experiment. The lower bound is predicted at $\lambda_{\operatorname{marg}} / a=1.08 \pm 0.03$, while the measurement gives $\lambda_{\min } / a=1.13 \pm 0.03$. In a similar experiment [6], the lower bound of the stable band was found to coincide with the final ripple length: $\lambda_{\min }=\tilde{\lambda}$, whereas we find that $\lambda_{\min }<\tilde{\lambda}$. The difference between the two results might be apparent only, as the number of ripples in the annulus of [6] was 10 or smaller, giving rise to an uncertainty in the ripple length on the order of the difference between $\lambda_{\min }$ and $\tilde{\lambda}$.

The dynamics responsible for the evolution of a 1D ripple pattern are also relevant for $2 \mathrm{D}$ ripple patterns. However, in the latter case, topological defects will be present [7], and the final length selection might be determined by the motion of these [9].

It is a pleasure to acknowledge discussions with T. Bohr, M. van Hecke, and F. Schmidt. J. K. is grateful to DTU and NBI for gracious hospitality, and to DFG within SFB237 for support. K. H. A thanks Universität Essen for hospitality.

[1] M. C. Cross and P. C. Hohenberg, Rev. Mod. Phys. 65, 851 (1993), and references therein.

[2] H. Nishimori and N. Ouchi, Phys. Rev. Lett. 71, 197 (1993).

[3] B. T. Werner and D. T. Gillespie, Phys. Rev. Lett. 71, 3230-3233 (1993).

[4] Z. Csahók, C. Misbah, F. Rioual, and A. Valance, Eur. Phys. J. E 3, 71 (2000).

[5] A. Betat, V. Frette, and I. Rehberg, Phys. Rev. Lett. 83, 88-91 (1999).

[6] A. Stegner and J.E. Wesfreid, Phys. Rev. E 60, R3487 (1999).

[7] J. L. Hansen, M. v. Hecke, A. Haaning, C. Ellegaard, K. H. Andersen, T. Bohr, and T. Sams, Nature (London) 410, 324 (2001); J. L. Hansen, M. v. Hecke, C. Ellegaard, K. H. Andersen, T. Bohr, and T. Sams, Phys. Rev. Lett. 87, 204301 (2001).

[8] R. A. Bagnold, Proc. R. Soc. London A 187, 1-15 (1946).

[9] K. H. Andersen, M.-L. Chabanol, and M. v. Hecke, Phys. Rev. E 63, 066308 (2001).

[10] M. A. Scherer, F. Melo, and M. Marder, Phys. Fluids 11, 58 (1999).

[11] P. Nielsen, J. Geophys. Res. 86, 6467 (1981).

[12] P. Blondeaux, J. Fluid Mech. 218, 1-17 (1990); K. H. Andersen, Phys. Fluids 13, 58-64 (2001).

[13] We consider here initial conditions with some amount of disorder. Different behavior results in the (unrealistic) case of a perfectly ordered state with a localized perturbation; see J. Krug, Advances in Complex Systems (to be published).

[14] L. Breiman and J. H. Friedman, J. Am. Stat. Assoc. 80, 580-598 (1985).

[15] H. Voss, P. Kolodner, M. Abel, and J. Kurths, Phys. Rev. Lett. 83, 3422-3425 (1999).

[16] For example, for $f_{l}$ : $\psi_{l}=\left\langle f_{l}(t) \cdot S(t)\right\rangle /\left(\left\langle f_{l}^{2}\right\rangle\left\langle S^{2}\right\rangle\right)$; $S(t)=\dot{\lambda}_{i}-2 f_{c}+f_{r}$ has zero mean (without loss of generality). 